\title{
Morphology-Induced Information Transfer in Bat Sonar
}

\author{
Jonas Reijniers \\ Departement Biologie, Universiteit Antwerpen, B-2020 Antwerpen, Belgium
}

Dieter Vanderelst and Herbert Peremans

Departement MTT, Universiteit Antwerpen, B-2000 Antwerpen, Belgium

(Received 10 December 2009; revised manuscript received 25 August 2010; published 30 September 2010)

\begin{abstract}
It has been argued that an important part of understanding bat echolocation comes down to understanding the morphology of the bat sound processing apparatus. In this Letter we present a method based on information theory that allows us to assess target localization performance of bat sonar, without a priori knowledge on the position, size, or shape of the reflecting target. We demonstrate this method using simulated directivity patterns of the frequency-modulated bat Micronycteris microtis. The results of this analysis indicate that the morphology of this bat's sound processing apparatus has evolved to be a compromise between sensitivity and accuracy with the pinnae and the noseleaf playing different roles.
\end{abstract}

DOI: 10.1103/PhysRevLett.105.148701

PACS numbers: $43.80 .+\mathrm{p}, 43.60 .+\mathrm{d}$

Bats, similar to other mammals, are known to rely on spectral cues introduced by the interaction of the impinging sound field with their head and outer ears to localize objects [1]. In the case of bats, this direction dependent reception filter, the so-called head related transfer function (HRTF), is complemented by direction dependent sound emission [2,3] as echolocation is an active sensing modality. Because of the important contribution made by this acoustic preprocessing in the physical domain to the final sound localization capabilities of bats, it is to be expected that, throughout years of evolution, the morphology of this sound processing apparatus has been optimized accordingly.

Previous attempts at relating morphology to echolocation performance have been based on qualitative analyses of salient features in the HRTF, e.g., the presence of spectral peaks and notches $[2,4]$. However, there is a need for a more quantitative information measure, which would allow us to understand how the sonar system was optimized during evolution.

Altes [5] was the first to set out such a quantitative measure, based on a maximum likelihood analysis of spectrum based localization. Recently, Müller et al. [6] made use of these results to study the significance of monaural spectral position cues introduced by the tragus into the HRTF of the brown long-eared bat. However, these analyses suffer from two important limitations. First, the proposed noise models do not take into account unknown reflector filtering and include additive system noise only. Consequently, the results indicate that an arbitrarily high angular accuracy can be obtained for sufficiently strong echoes. However, in reality even high intensity echoes show nonzero deviations from the expected echo spectra due to reflector filtering. Second, the emitter is not taken into account, hereby precluding the study of how the pinna and noseleaf directionalities interact to provide target location cues for the bat sonar system. In this Letter we present a new method, based on an information theoretic analysis of spectrum based object localization that addresses these limitations.

The model used in this Letter is shown in Fig. 1. It views the environment as a source producing symbols $\theta$, each symbol corresponding with a particular target direction. The noseleaf and pinnae taken together are considered to act as the encoder that transforms the symbol $\theta$ into a signal (template $T_{\theta}$ ) that can be transmitted through the channel. Channel distortions in the model consist of both additive system noise and unknown reflector filtering. Finally, the mutual information between the received echo spectrum and the target position is proposed as the relevant global performance measure.

To calculate the HRTF, $H_{\boldsymbol{\theta}}^{\mathrm{hrtf}}(f)$, we use the procedure described in Ref. [7]. First, a 3D computer model of the bat head is constructed by scanning a Micronycteris microtis head in a microCT machine, see Fig. 1. Next, the HRTF is calculated using BEM3D software. A sound source with a flat response over the frequency range of interest is positioned at different azimuths and elevations $(\boldsymbol{\theta})$ in the far field. The

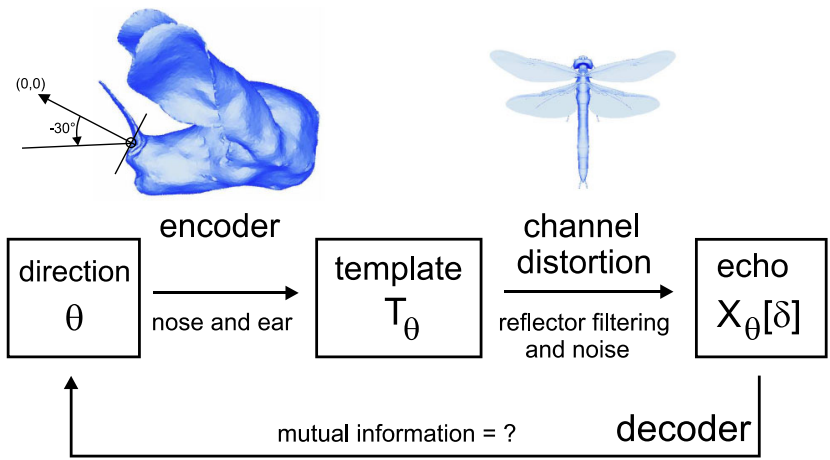

FIG. 1 (color online). Noise channel model of the information transfer in bat sonar. 
HRTF $H_{\boldsymbol{\theta}}^{\text {ears }}(f)$ is then given by the simulated sound amplitudes at the entrances of both ear canals. We take the binaural spectrum to be the concatenation of the two monaural spectra [5]. The same software is used to simulate the emission pattern of the noseleaf $H_{\boldsymbol{\theta}}^{\text {nose }}(f)$, except now, the emitters are positioned in the nostril openings, and the receivers are positioned in the far field at the same azimuths and elevations used for the HRTF calculation. Note that for the emitter beam pattern, the power integrated over all directions is normalized to 1 for all frequencies, as we assume that the power spectral density of the emitted sound is constant for all frequencies in the active frequency range. As an alternative emitter configuration, we also consider the extreme case where the emitter is a point source emitting equal power in all directions $H_{\boldsymbol{\theta}}^{\text {point }}$.

As mentioned before, we include both system noise and reflector filtering in the channel model. Their different effects on the echo spectra follow in a rather natural way if we represent the received spectra on a logarithmic scale, i.e., perform a compression very similar to the one performed by the hearing apparatus [8]. Hence, all spectral variables $(\boldsymbol{X}, \boldsymbol{H}, \ldots)$ used below are on a logarithmic scale (in $\mathrm{dB}$ ). System noise is additive but, because of the logarithmic compression, its effect on the spectrum can be approximated by a maximum operator. In this approximation, the noise power spectral density $N_{0}$ defines the lower threshold below which no signal can be detected. Hence the echo spectrum $\boldsymbol{X}_{\boldsymbol{\theta}}^{\text {refl }}$ reflected from direction $\boldsymbol{\theta}$, originally emitted by a nose with emission pattern $\boldsymbol{H}_{\boldsymbol{\theta}}^{\text {nose }}$, undergoing an unknown filter operation during reflection $\boldsymbol{H}^{\text {refl }}$, and picked up by ears with HRTF $\boldsymbol{H}_{\boldsymbol{\theta}}^{\text {ears }}$, can be approximated by

$$
\boldsymbol{X}_{\boldsymbol{\theta}}^{\text {refl }} \approx \max \left(\boldsymbol{H}_{\boldsymbol{\theta}}^{\text {nose }}+\boldsymbol{H}^{\text {refl }}+\boldsymbol{H}_{\boldsymbol{\theta}}^{\text {ears }}, N_{0}\right) .
$$

Note that, in the absence of specific information, we assume the noise power spectral density $N_{0}$ to be constant for the frequency range of interest. We can rewrite the unknown filter operation introduced by the reflector as $\boldsymbol{H}^{\text {refl }}=\alpha+\boldsymbol{\delta}$, with $\alpha=\left\langle H^{\text {refl }}\right\rangle_{f}$ the strength of the reflection averaged over all frequencies, and $\boldsymbol{\delta}$ the spectral error introduced by this reflector filtering with zero average $\langle\boldsymbol{\delta}\rangle_{f}=0$ over all frequencies. Substituting this in Eq. (1) then yields

$$
\begin{aligned}
\boldsymbol{X}_{\boldsymbol{\theta}}^{\text {refl }} & =\boldsymbol{X}_{\boldsymbol{\theta}}^{\alpha}[\boldsymbol{\delta}] \\
& \approx \max \left\{\alpha+\boldsymbol{H}_{\boldsymbol{\theta}}^{\text {nose }}+\boldsymbol{H}_{\boldsymbol{\theta}}^{\text {ears }}, N_{0}\right\}+\boldsymbol{\delta} \\
& =\boldsymbol{T}_{\boldsymbol{\theta}}^{\alpha}+\boldsymbol{\delta},
\end{aligned}
$$

where we define $\boldsymbol{T}_{\boldsymbol{\theta}}^{\alpha}$ as the binaural spectral template corresponding to reflection strength $\alpha$ and direction $\boldsymbol{\theta}$. This approximation (see Ref. [9]) allows us to consider the errors introduced by the unknown reflector filter operation $\boldsymbol{\delta}$ as additive "noise" on the spectral template $\boldsymbol{T}_{\boldsymbol{\theta}}^{\alpha}$. Based on empirical data from realistic reflectors, we model this filter "noise" by a multivariate Gaussian distribution with mean $\langle\boldsymbol{\delta}\rangle_{f}=0$ and covariance matrix

$$
\Sigma=\sigma^{2}\left[\begin{array}{cc}
\boldsymbol{A} & \gamma \boldsymbol{A} \\
\gamma \boldsymbol{A} & \boldsymbol{A}
\end{array}\right]
$$

with $A(i, j)=\exp \left[-(i-j)^{2} /\left(2 \sigma_{f}^{2}\right)\right]$, where $\sigma_{f}$ (in $\mathrm{kHz}$ ) is a measure for the correlation length of the reflector filtering along the frequency axis, see Ref. [9]. The variance $\sigma^{2}$ introduced by the reflector filtering is identical for all frequencies and correlated in both ears with a factor $\gamma$.

The information $I(\Theta ; X)$ any measured spectrum $X$ carries on the direction $\boldsymbol{\theta}$ of the reflector can be written [10] as the reduction in the entropy of $\boldsymbol{\theta}$, given $\boldsymbol{X}$, i.e.,

$$
\begin{aligned}
I(\Theta ; \boldsymbol{X}) & =H(\Theta)-H(\Theta \mid \boldsymbol{X}) \\
& =H(\Theta)+\int_{\boldsymbol{\theta}} d \boldsymbol{\theta} P(\boldsymbol{\theta} \mid \boldsymbol{X}) \log _{2}[P(\boldsymbol{\theta} \mid \boldsymbol{X})],
\end{aligned}
$$

where $\boldsymbol{\theta}$ runs over all realizations of the stochastic variable $\Theta, H(\Theta)$ denotes the entropy of $\boldsymbol{\theta}$ and $H(\Theta \mid X)$ is the conditional entropy. $I(\Theta ; \boldsymbol{X})$ is expressed in bits. In order to calculate $P(\boldsymbol{\theta} \mid \boldsymbol{X})$, we make use of Bayes Rule

$$
P(\boldsymbol{\theta} \mid \boldsymbol{X})=\frac{P(\boldsymbol{\theta}) P(\boldsymbol{X} \mid \boldsymbol{\theta})}{P(\boldsymbol{X})} .
$$

As we assume no prior knowledge on $\boldsymbol{\theta}, P(\boldsymbol{\theta})$ is uniformly distributed. Since we also presuppose no prior knowledge on the reflector, we do not know $\alpha$ and consequently the conditional probability for spectrum $\boldsymbol{X}$ given $\boldsymbol{\theta}$ can be written as

$$
P(\boldsymbol{X} \mid \boldsymbol{\theta})=\int_{\alpha} d \alpha P(\alpha) P(\boldsymbol{X} \mid \boldsymbol{\theta}, \alpha) .
$$

In this expression $P(\boldsymbol{X} \mid \boldsymbol{\theta}, \alpha)$ can be interpreted as the probability that the difference between $\boldsymbol{X}$ and $\boldsymbol{T}_{\boldsymbol{\theta}}^{\alpha}$, is due to environmental effects,

$$
P(\boldsymbol{X} \mid \boldsymbol{\theta}, \alpha)=\frac{1}{(2 \pi)^{N / 2}|\mathbf{\Sigma}(\boldsymbol{X})|^{1 / 2}} e^{-1 / 2\left(\boldsymbol{X}-\boldsymbol{T}_{\boldsymbol{\theta}}^{\alpha}\right) \mathbf{\Sigma}(\boldsymbol{X})^{-1}\left(\boldsymbol{X}-\boldsymbol{T}_{\boldsymbol{\theta}}^{\alpha}\right)},
$$

with the covariance matrix $\boldsymbol{\Sigma}(\boldsymbol{X})=\boldsymbol{\Sigma}$ except for frequencies where the echo spectrum $\boldsymbol{X}$ is below the noise level and the correlation is set to zero, see Ref. [9]. Finally, $P(\boldsymbol{\theta} \mid \boldsymbol{X})$ can then be obtained by simple normalization. This result allows us to calculate the information $I(\Theta ; X)$ for any particular spectrum $\boldsymbol{X}$. The quantity of direct behavioral relevance though is the average information carried by all possible spectra originating from direction $\boldsymbol{\theta}$ with strength $\alpha$. To calculate this quantity one should average over all realizations of the reflector filter ensemble, i.e.,

$$
\left\langle I\left(\Theta ; \boldsymbol{X}_{\boldsymbol{\theta}}^{\alpha}[\boldsymbol{\delta}]\right)\right\rangle_{\boldsymbol{\delta}}=\int[d \boldsymbol{X}] P(\boldsymbol{X} \mid \boldsymbol{\theta}, \alpha) I(\Theta ; \boldsymbol{X}) .
$$

As Eq. (10) cannot be calculated directly we use a Monte Carlo approximation. The number of random filter realizations was chosen such that for all directions $\boldsymbol{\theta}$ the standard deviation is smaller than 0.5 bits.

Note that the directional information $I_{\boldsymbol{\theta}}^{\alpha}=$ $\left\langle I\left(\Theta ; \boldsymbol{X}_{\boldsymbol{\theta}}^{\alpha}[\boldsymbol{\delta}]\right)\right\rangle_{\boldsymbol{\delta}}$ can easily be linked to other relevant quantities such as the solid angle $\Omega_{\boldsymbol{\theta}}^{\alpha}=2^{1-I_{\boldsymbol{\theta}}^{\alpha}} \pi$, which is a 
measure for the area of confusion (accuracy) surrounding a target with reflection strength $\alpha$ at a particular position $\boldsymbol{\theta}$.

To calculate numerical values for this directional information given a specific nose or ear system, we consider the full active frequency range [55-150] $\mathrm{kHz}$ (see Ref. [11]) in steps of $\Delta f=1 \mathrm{kHz}$. We sample $\boldsymbol{\theta}$ uniformly over the frontal hemisphere, so that every $\boldsymbol{\theta}_{i}$ corresponds to a similar solid angle $2 \pi / n$, with $n=2352$ the number of possible different directions (corresponding to $\Delta \boldsymbol{\theta}=3^{\circ}$ ). We assume a uniformly distributed reflection strength $\alpha=$ $[0,160] \mathrm{dB}$ which is discretized with stepsize $\Delta \alpha=$ $2.5 \mathrm{~dB}$. The correlation between left and right ear was set to $\gamma=0.95 ; \sigma_{f}$ was set to $2 \mathrm{kHz}$. In Fig. 2 we have shown the directional information for the pinnae in combination with a point source emitter (a-e) and with the noseleaf $(\mathrm{f}-\mathrm{j})$, for different reflection strengths $\alpha$. When the reflection strength $\alpha$ is low, the additive noise corrupts the signal to such a degree that the echo does not contain any information about the reflector position. As $\alpha$ increases, there are certain subregions from the frontal hemisphere where the echo signal rises above the noise and starts carrying position information, see Figs. 2(a) and 2(f). As $\alpha$ further increases, the information per echo increases along until it starts saturating for echoes coming from the most sensitive frontal area, see Figs. 2(c) and 2(h). For those reflector positions the echo spectrum is well above the system noise level which no longer limits the information transfer. However, unknown reflector filtering now limits the maximum information that can be carried by these echo signals. At the same time, increasing numbers of peripheral echo signals rise above the noise and start carrying information. Eventually, the information attains its maximum, as all spectra are well above noise level.

We notice that for $0^{\circ}$ azimuth, the information content is reduced for most elevations. This can be understood by realizing that both the spectral templates as well as the reflector filtering $(\gamma \approx 1)$ for both ears are nearly identical for azimuth $0^{\circ}$. Hence, it is easy to discriminate azimuth $0^{\circ}$ by checking whether the received left and right echo spectra are identical. However, to estimate the elevation angle in the midsaggital plane, one can only rely on spectral cues and those are distorted most by the unknown reflector filtering. The resulting ambiguity [12] along the target elevation axis causes the reduction of the information.

If we compare the directional information for both emitter configurations, we arrive at the following observations regarding the different roles played by the pinnae and the noseleaf in the generation of spatial information. From the comparison of Figs. 2(e) and 2(j), we conclude that the spectral cues necessary for spatial localization are predominantly provided by the pinnae. Indeed, including the noseleaf introduces very little additional location cues into the echo spectra, as indicated by the similarity of both plots. It seems the noseleaf mostly affects the echo strength and thus the reflector strength $\alpha$ at which these localization cues rise above the noise level. As is clear from Figs. 2(c), 2(d), 2(h), and 2(i), the combination with the noseleaf is more sensitive to weak reflectors (small $\alpha$ ) from the frontal region, but less sensitive to echoes returning from the periphery. This is due to the noseleaf directing sound towards the frontal region, thereby increasing the echo strength and thus the capacity of echo signals from that region to carry position information, at the expense of the periphery.

With regard to the spatial information introduced by the pinnae, there seems to be a trade-off between sensitivity and accuracy. This can be inferred from comparing Figs. 2(b) and 2(c), when the noseleaf is not taken into account. The high informational subregion (darker colors) in Fig. 2(c) is rather insensitive, as echoes originating from this region do not yet carry information in Fig. 2(b). On the other hand, more sensitive directions that already carry information in Fig. 2(b) correspond with the intermediate accuracy regions in Fig. 2(c). This trade-off can be understood by noting the distinct spectral notch at $75 \mathrm{kHz}$ in Fig. 2(k) which coincides with this low sensitivity and high accuracy subregion. This suggests that the accurate angular
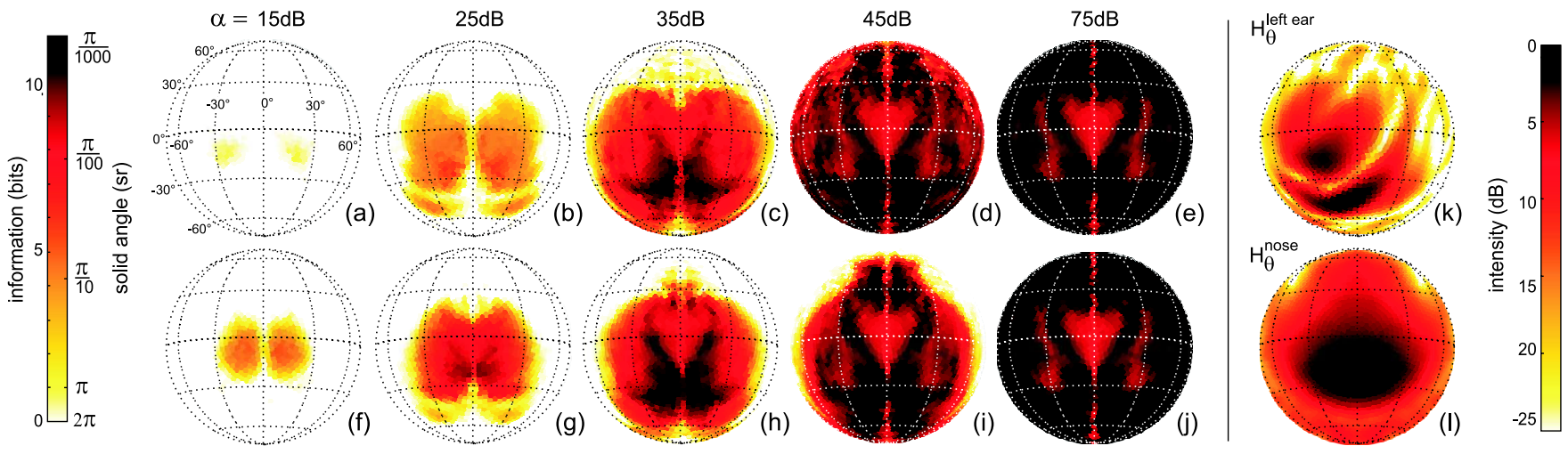

FIG. 2 (color online). (left) The directional information $\left\langle I\left(\Theta ; \boldsymbol{X}_{\boldsymbol{\theta}}^{\alpha}[\boldsymbol{\delta}]\right)\right\rangle_{\boldsymbol{\delta}}$ for the ear in combination with point source (a)-(e) and noseleaf emitter (f)-(j), for different reflection strengths $\alpha$ with $\sigma=10 \mathrm{~dB}, \gamma=0.95, \sigma_{f}=2 \mathrm{kHz}$. The correspondence between amount of information and area of confusion is also indicated. (right) The left ear $(k)$ and nose $(l)$ directivity at $75 \mathrm{kHz}$. 


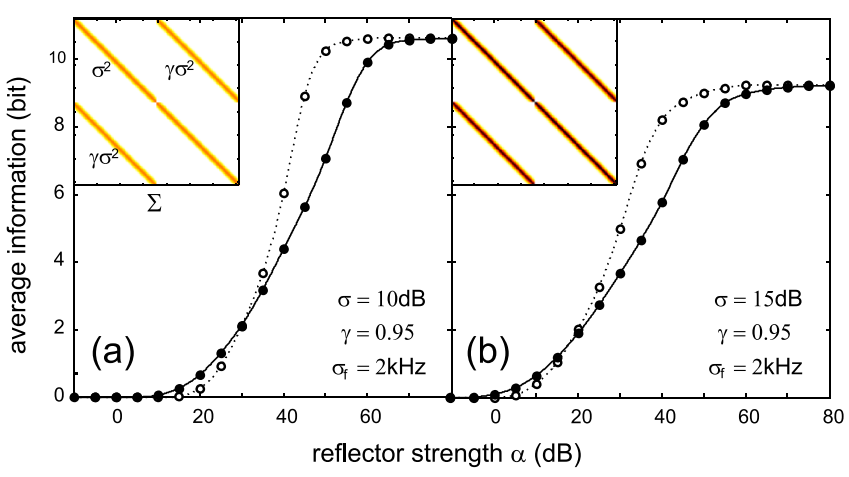

FIG. 3 (color online). The directional information averaged over all directions as a function of the reflector strength $\alpha$, for two different reflector filtering distributions $\sigma=10,15 \mathrm{~dB}$. Full circles correspond to the ear-noseleaf combination, hollow circles to the ear-point source emitter. The inset shows the corresponding covariance matrices.

information is mainly encoded by this spectral notch, which shifts as a function of frequency. However, as spectral notch encoding operates by selectively removing sound energy, accuracy comes at the price of sensitivity loss.

But if we look at Fig. 2(1), it seems that the noseleaf compensates for the reduced sensitivity in this region of interest. By directing the sound into a region corresponding with high accuracy but low sensitivity, it improves the sensitivity in this region while avoiding the cost of accuracy loss, as can be inferred from comparing Figs. 2(b) and 2(c) with Figs. 2(g) and 2(h). This optimal region is centered around azimuth $0^{\circ}$ and starts at elevation $-20^{\circ}$ to expand downward for increasing $\alpha$, centering around $-30^{\circ}$. As is clear from Figs. 2(g) and 2(h), already at low reflection strengths, the echoes returning from this area are highly informational and allow accurate localization.

The influence of the noseleaf on the overall echolocation performance is shown in Fig. 3, where we plotted the directional information averaged over all directions as a function of reflection strength $\alpha$ for $\sigma=10 \mathrm{~dB}$ (left), for both emitter configurations. This average directional information can be considered to be the quality measure of the nose and ear system for localizing reflectors with reflection strength $\alpha$. For lower $\alpha$ values we conclude that the presence of the noseleaf improves the average information contained within the echo signals. At $\alpha \approx 30 \mathrm{~dB}$ both performance curves intersect and the configuration with the point source emitter starts outperforming the noseleaf configuration. This indicates that the advantages of the sound redirection due to the noseleaf come at the cost of a reduced information transfer for echoes of intermediate strength in the periphery, see Figs. 2(d) and 2(i). Finally, for higher $\alpha$ values the average information attains a maximum when all echo spectra are well above noise level. Further increases in the reflector strength $\alpha$ do not result in improved information extraction as unknown reflector filtering is now the limiting factor. Similar results are obtained for different reflector filtering strengths, e.g., $\sigma=15 \mathrm{~dB}$, Fig. 3 (right), except that as $\sigma$ increases, the average information decreases as expected, since the signal is increasingly distorted by reflector filtering. Changing other reflector filtering characteristics $\left(\gamma, \sigma_{f}\right)$ also changes the results quantitatively but not qualitatively, see Ref. [9].

From this analysis of the echolocation system of the noseleaved bat Micronycteris microtis we conclude that the morphology of the nose and ear system is driven by a trade-off between sensitivity and accuracy. A similar sensitivity-accuracy trade-off was described recently in [13] for the biosonar of Egyptian fruit bats. We also conclude that the noseleaf and the pinnae play different roles. The pinnae mainly introduce spatially dependent spectral variations whereas the noseleaf contribution mainly consists of selectively insonifying relevant frontal areas at the expense of the periphery of the frontal hemisphere. Note that these conclusions all follow from the hypothesis that the combined ear-nose morphology has evolved to reliably encode target position in spectral cues despite spectral distortions due to additive noise and unknown reflector filtering. As the analysis presented here provides direct predictions about the spatial distribution of accuracy and sensitivity that can be readily tested with behavioral experiments the validity of this hypothesis can be straightforwardly ascertained.

We thank the reviewers for their constructive comments. This work was funded through the CILIA and ChiRoPing projects (EU - FET). Bat specimen provided by I. Geipel and E. Kalko, University of Ulm, Germany.

[1] B. D. Lawrence and J.A. Simmons, Science 218, 481 (1982).

[2] J. M. Wotton, R. L. Jenison, and D. J. Hartley, J. Acoust. Soc. Am. 101, 1723 (1997).

[3] H.-U. Schnitzler and A. D. Grinnell, J. Comp. Physiol. 116, 51 (1977).

[4] M. K. Obrist, M. B. Fenton, J. L. Eger, and P. A. Schlegel, J. Exp. Biol. 180, 119 (1993).

[5] R. A. Altes, J. Acoust. Soc. Am. 63, 155 (1978).

[6] R. Müller, H. Lu, and J. R. Buck, Phys. Rev. Lett. 100, 108701 (2008).

[7] F. De Mey, J. Reijniers, H. Peremans, M. Otani, and U. Firzlaff, J. Acoust. Soc. Am. 124, 2123 (2008).

[8] L. Wiegrebe, Biol. Cybern. 98, 587 (2008).

[9] See supplementary material at http://link.aps.org/ supplemental/10.1103/PhysRevLett.105.148701.

[10] T. M. Cover and J. A. Thomas, Elements of Information Theory (John Wiley \& Sons, New York, NY, 1991).

[11] D. Vanderelst, F. De Mey, H. Peremans, I. Geipel, E. Kalko, and U. Firzlaff, PLoS ONE 5, e11893 (2010).

[12] J. C. Makous and J. C. Middlebrooks, J. Acoust. Soc. Am. 87, 2188 (1990).

[13] Y. Yovel, B. Falk, C. Moss, and N. Ulanovsky, Science 327, 701 (2010). 